\title{
Effect of Large-Angle Scattering between Ions and Neutral Particles on the Density Profile at the Divertor Plate ${ }^{*}$
}

\author{
Daisuke UMEZAKI and Hideaki MATSUURA \\ Department of Applied Quantum Physics and Nuclear Engineering, \\ Kyushu University, 744 Motooka, Fukuoka 819-0395, Japan
}

(Received 16 November 2020 / Accepted 14 January 2021)

\begin{abstract}
The better radial transport modelling of the SOL/divertor plasmas is required to obtain quantitative agreements between experimental values and simulation results towards the proper SOL/divertor plasma prediction of the future magnetic fusion devices. Various SOL/divertor codes (e.g. SONIC, SOLPS-ITER, EDGE2D, UEDGE, EMC3-Eirene, etc.) have been developed and applied to the interpretative simulations of the present fusion devices. Above codes treats the radial transport as a diffusion model and there exists uncertainty to decide the value of the diffusion coefficients. The simulation by the SONIC has resulted in a few times larger ion flux towards the divertor plate than the experimental value observed in JT-60U possibly due to such an uncertainty of the diffusion process. To achieve the quantitative agreement between the experiments and the simulation results is indispensable to predict SOL/divertor plasma parameters of the future fusion devices. Here, radial transport was simulated by considering large-angle elastic scattering between ions and neutral particles and by applying the probability distribution function to fluid equations in the divertor plasma. It was found that the density peak and ion flux are reduced by about half.
\end{abstract}

(C) 2021 The Japan Society of Plasma Science and Nuclear Fusion Research

Keywords: divertor, edge plasma, SOL, large-angle scattering, elastic scattering

DOI: $10.1585 /$ pfr. 16.2403021

\section{Introduction}

Various SOL/divertor codes have been developed for the design and prediction of fusion devices, including SOLPS [1-3], SONIC [4, 5], EDGE2D [6], UEDGE [7], and EMC3-Eirene [8]. Although these codes have been very successful, the simulation of the JT-60U experimentusing the SONIC code has overestimated the ion flux at the strike pointby a factor of two or more with respect to the experimental value [9]. Some other important quantities have also not been reproduced correctly, from a quantitative point of view. In particular, the radial transport mechanism in the edge plasma has remained unclear, and the experimentally observed diffusion coefficient is about one order of magnitude larger than the theoretically predicted value; hence, this is treated as an anomalous diffusion in the above codes. The radial transport can affect some important quantities, thus influencing the final prediction results. Some models (e.g., [10]) have been proposed to describe the anomalous transport in the SOL.

On the other hand, in edge plasma, neutral particles are generated by recombination, neutralization on the wall, and gas puffing. A neutral particle flux of amplitude equal to or higher than the ion flux has been observed in JT$60 \mathrm{U}$ [11]. Elastic scattering between ions and neutral particles can be a large-angle scattering process, and the scat-

author'se-mail: d_umezaki@nucl.kyushu-u.ac.jp

*) This article is based on the presentation at the 29th International Toki Conference on Plasma and Fusion Research (ITC29). tered particle flight direction can be changed significantly in a single scattering event compared with the Coulomb scattering. Figure 1 shows the elastic differential scattering cross sections between deuteron and deuterium [12].

In this work, the probability distribution function [13, 14] (described in section 2.4) was applied to fluid equations forthe plasma, and the transport driven by largeangle elastic scattering between ions and neutral particles was studied including the radial direction. Furthermore,

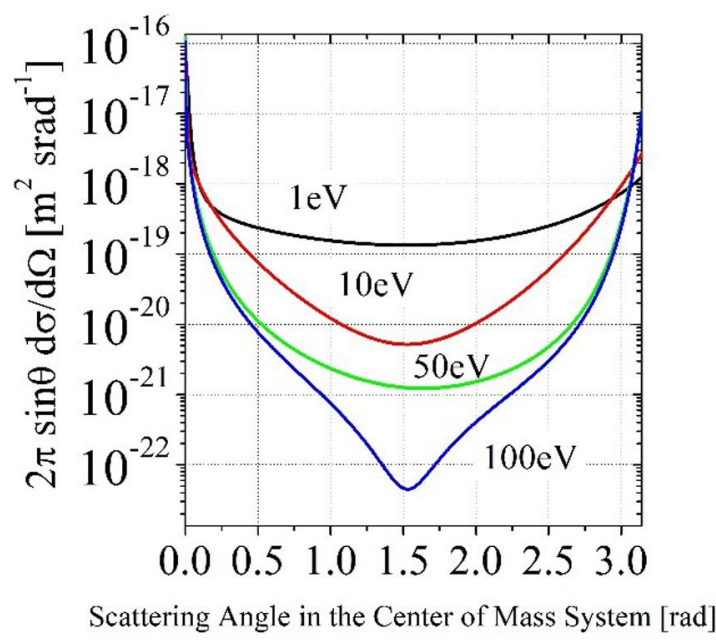

Fig. 1 Elastic differential scattering cross section between deuteron and deuterium [12]. 
the influence on the density profile and ion flux at the divertor plate was investigated.

\section{Analysis Model}

\subsection{Calculation scheme and flow}

A two-dimensional square scheme $\left(25 \times 25 \mathrm{~cm}^{2}\right)$ was implemented to model the divertor plasma region of JT$60 \mathrm{U}$ (Fig. 2). The number of numerical cells was setto 10000. Boundary conditions (described in section 2.2) were adopted except for the upstream, and the upstream boundary condition was provided based on the experimental value [9].

The calculation flow consists of the following four steps, as also shown in Fig. 3.

(0) Initial values of $n_{i}, \vec{u}_{l}, T_{i}$, and $T_{e}$ are input. The constant values, such as the diffusion coefficient $D$, are also input.

(1) The neutral particles density $n_{n}$ and temperature $T_{n}$ are calculated by relating to the plasma density $n_{i}$ and ion temperature $T_{i}$ (described in section 2.4). The coefficients determined from the plasma variables, such as the thermal conductivity coefficient, are calculated for the fluid equations.

(2) The source terms of the fluid equations are computed. In the case of the EL model, $S_{E L}, \overrightarrow{S_{P_{E L}}}$, and $S_{i_{E L}}$ are computed.

(3) The fluid equations are solved,thus permitting to obtain $n_{i}, \vec{u}_{l}, T_{i}$, and $T_{e}$. In the case of the diffusion model, the radial velocity $u_{y}$ is computed through the diffusion equation.

(4) The possible calculation convergence is evaluated. The residual sums of squares (RSS) of $n_{i}, T_{i}$, and $T_{e}$ between the current and previous steps are computed in all the control volumes. The calculation is considered to be finished when the RSS average in all control volumes is smaller than $10^{-15}$, otherwise the calculation flow is repeated starting from step (1).

\subsection{Fluid equations}

2D fluid equations were used for the plasma. These equations are identical to those in the original B2-code [15], and are based on Braginskii equations [16]. The fluid equations can be written as:

$$
\begin{aligned}
& \frac{\partial \rho_{i}}{\partial t}+\nabla \cdot\left(\rho_{i} \vec{u}\right)=S_{\rho_{i}} \\
& \frac{\partial}{\partial t}\left(\rho_{i} \vec{u}\right)+\nabla \cdot\left(\rho_{i} \vec{u} \vec{u}\right)=-\nabla\left(P_{i}+P_{e}\right)-\nabla \cdot \overleftrightarrow{\Pi}+\overrightarrow{S_{p}}
\end{aligned}
$$

$$
\begin{aligned}
\frac{\partial}{\partial t}( & \left.\frac{3}{2} n_{i} T_{i}+\frac{1}{2} n_{i} m u^{2}\right)+\nabla \\
\cdot & {\left[\vec{q}+\left(\frac{5}{2} n_{i} T_{i}+\frac{1}{2} n m_{i} u^{2}\right) \vec{u}+\vec{\Pi} \cdot \vec{u}\right] } \\
= & \vec{u} \cdot \nabla P_{e}-n_{e} v\left(T_{i}-T_{e}\right)+S_{i},
\end{aligned}
$$

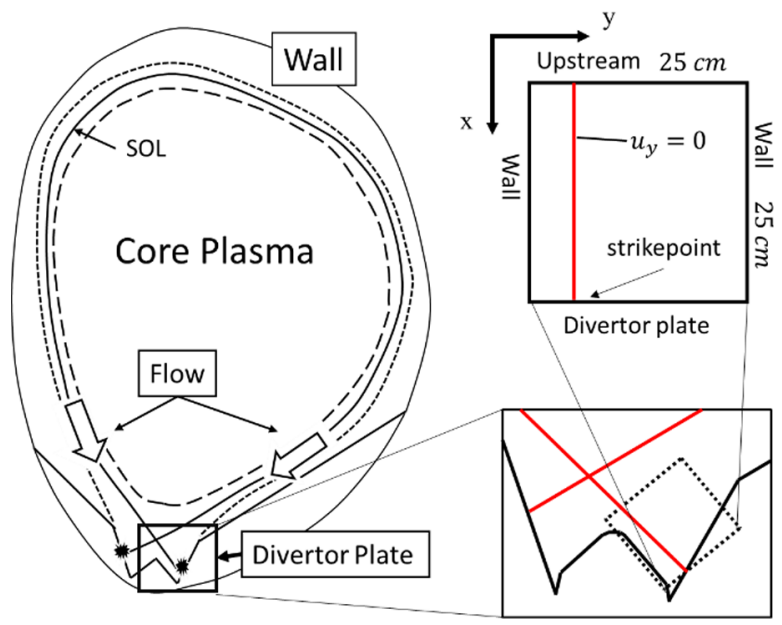

Fig. 2 Calculation scheme with reference to the divertor plasma region of JT-60U.

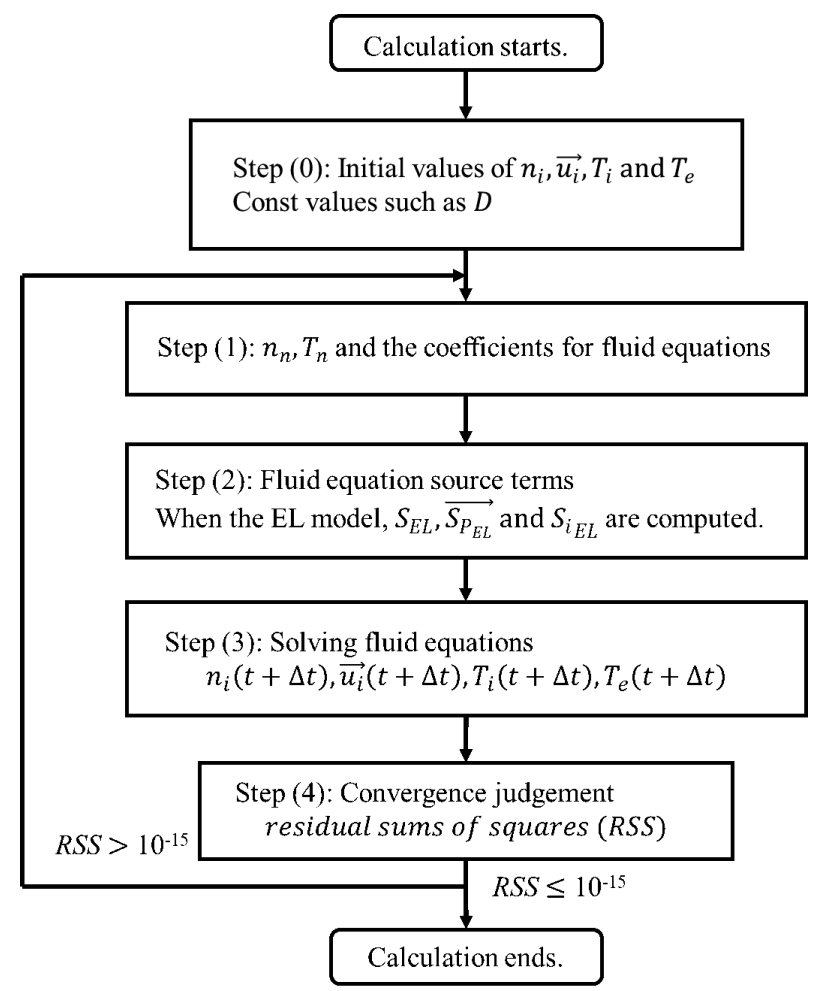

Fig. 3 Calculation flow.

$$
\begin{gathered}
\frac{\partial}{\partial t}\left(\frac{3}{2} n_{e} T_{e}\right)+\nabla \cdot\left[\frac{5}{2} n_{e} T_{e} \vec{u}-k_{e} \nabla T_{e}\right] \\
=\vec{u} \cdot \nabla P_{e}-n_{e} v\left(T_{e}-T_{i}\right)+S_{e} .
\end{gathered}
$$

Here, $\vec{u}=\left(u_{x}, u_{y}\right)$ is the velocity, $n(\rho)$ is the (mass) density, $T_{i}, T_{e}, P_{i}$, and $P_{e}$ are the ion and electron temperature, and the ion and electron pressure, respectively. $S_{\rho_{i}}, \overrightarrow{S_{p}}, S_{i}$, and $S_{e}$ are the source terms, which are written according to:

$$
\begin{aligned}
& S_{\rho_{i}}=n_{i} n_{e}\langle\sigma v\rangle_{I O N}-n_{e} n_{e}\langle\sigma v\rangle_{R C}+S_{E L} \\
& \overrightarrow{S_{p}}=m_{i} n_{n} n_{i}\left(\langle\sigma v\rangle_{C X}+\langle\sigma v\rangle_{I O N}\right) \cdot \overrightarrow{u_{n}}
\end{aligned}
$$




$$
\begin{aligned}
& -m_{i} n_{e} n_{e}\left(\langle\sigma v\rangle_{R C}+\langle\sigma v\rangle_{C X}\right) \cdot \overrightarrow{u_{l}}+\overrightarrow{S_{P E L},} \\
S_{i}= & n_{e} n_{i}\left(\langle\sigma v\rangle_{I O N}+\langle\sigma v\rangle_{C X}\right) \cdot E_{n} \\
& -n_{n} n_{i}\left(\langle\sigma v\rangle_{R C}+\langle\sigma v\rangle_{C X}\right) \cdot T_{i}+S_{i_{E L}}, \\
S_{e}= & -n_{n} n_{e}\langle\sigma v\rangle_{I O N} \cdot \delta_{e}-n_{e} n_{i}\langle\sigma v\rangle_{R C} \cdot T_{i} .
\end{aligned}
$$

$\langle\sigma v\rangle$ is the reaction rate coefficient. The effects of the elastic scattering $S_{E L}, \overrightarrow{S_{p_{E L}}}, S_{i_{E L}}$ are ignored in the diffusion model (described in section 2.3). The subscripts ION, RC, CX, and EL stand for ionization, recombination, charge exchange, and elastic scattering, respectively. $\overleftrightarrow{\Pi}=-\eta_{x} \nabla_{x} u_{x}-\eta_{y} \nabla_{y} u_{y}$ is the viscosity term, $n_{e} v\left(T_{i}-T_{e}\right)$ is the equi-partition term, and $\delta_{e}=25 \mathrm{eV}$.

The boundary conditions at the walls on both sides are as follows:

$$
\begin{aligned}
& \left.u_{x}\right|_{\text {wall }}=0, \\
& \left.u_{y}\right|_{\text {wall }}=a_{u_{y}} \sqrt{\frac{T_{i}+T_{e}}{m_{i}}}, \\
& \nabla_{y} n=\frac{n}{\lambda_{n}}, \\
& \nabla_{y} T_{i / e}=\frac{T_{i / e}}{\lambda_{T_{i / e}}},
\end{aligned}
$$

where $\lambda$ is the decay length of the ions and electrons. $\lambda_{n}$ and $\lambda_{T_{i / e}}$ were both set to $1 \mathrm{~cm} . \alpha_{u_{v}}$ is a freely-selected parameter and was here set to $10^{-3}$. The boundary conditions at the divertor plate are as follows [17]:

$$
\begin{aligned}
& \left.u_{x}\right|_{\text {diverotr }}=\sqrt{\frac{T_{i}+T_{e}}{m_{i}},} \\
& \nabla_{x} n=0, \\
& -k_{i x} \nabla_{x} T_{i}=\frac{3}{2} n T_{i} u_{x}, \\
& -k_{e x} \nabla_{x} T_{e}=4 n T_{e} u_{x} .
\end{aligned}
$$

Here, $k_{i x}=3.9 n_{i} T_{i} \tau_{i} / m_{i}$ and $k_{e x}=3.16 n_{e} T_{e} \tau_{e} / m_{e}$ are the ions and electrons parallel thermal conductivity coefficients, respectively. $\tau_{i}$ and $\tau_{e}$ are the ion and electron collision time, respectively.

\subsection{Diffusion model}

In this work, two models were computed for comparison. One model has also been used in the integrated SONIC code to obtain the radial velocity from the diffusion equation. This model is here denoted as the diffusion model. The diffusion equation is written as $u_{y}=$ $-D / n_{i} \cdot \nabla n_{i}$, where $D=0.3 \mathrm{~m}^{2} \mathrm{~s}^{-1}$ is the diffusion coefficient.

In the second model, ions are scattered by neutral particles and fly to other control volumes in various directions, including radial directions, to become a source. This model is here denoted as the EL model (described in section 2.4).

\subsection{Large-angle elastic scattering transport model: the EL model}

In this model, the ions are treated as a beam having velocity $\overrightarrow{v_{l}^{\prime}}$ equal to $\overrightarrow{u_{x}}$ and are scattered by neutral parti-

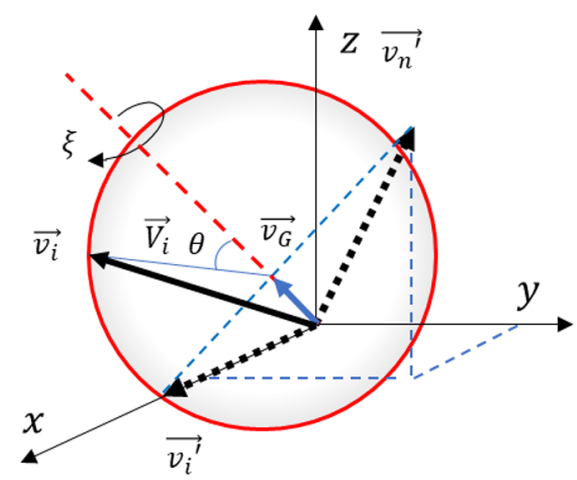

Fig. 4 Schematic view of the change in ion velocity before and after elastic scattering.

cles that have isotropic Maxwellian distribution. Figure 4 shows the change of the ion and neutral particle velocities before and after the elastic scattering. The scattered ion velocity is expressed by the probability distribution function $P\left(\overrightarrow{v_{l}^{\prime}} \rightarrow \overrightarrow{v_{l}} \mid \overrightarrow{v_{n}^{\prime}}\right)[13,14] . P$ represents the probability that an ion velocity changes from $\overrightarrow{v_{l}^{\prime}}$ to $\overrightarrow{v_{l}}$ as a result of a collision with a neutral particle that has velocity $\overrightarrow{v_{n}^{\prime}}$ in the laboratory system. In the velocity space, the scattered ion velocity $\overrightarrow{v_{l}}$ lies always on the surface of the sphere that has radius $\left|\overrightarrow{v_{n}^{\prime}}\right|$, with the center of the sphere being located at the end of the center-of-mass velocity $\overrightarrow{v_{G}}$. The neutral particle velocity $\overrightarrow{v_{n}{ }^{\prime}}$ is provided using random numbers. The value of the differential cross section $\theta d \sigma / d \Omega(\theta)$ is input into the velocity space cell on the sphere as $P\left(\overrightarrow{v_{l}^{\prime}} \rightarrow \overrightarrow{v_{l}} \mid \overrightarrow{v_{n}^{\prime}}\right)$, and $P$ is then normalized so that its integrated value becomes one. The scattered particle velocity distribution per unit time $Q\left(\vec{v}_{l}\right)$ is written as:

$$
\begin{aligned}
Q\left(\vec{v}_{l}\right)= & \iint \sigma_{E L,}\left(v_{r}\right) v_{r} P\left(\overrightarrow{v_{l}^{\prime}} \rightarrow \overrightarrow{v_{l}} \mid \overrightarrow{v_{n}^{\prime}}\right) \\
& \times f_{i}\left(\overrightarrow{v_{l}^{\prime}}\right) f_{n}\left(\overrightarrow{v_{n}^{\prime}}\right) d \overrightarrow{v_{l}^{\prime}} d \overrightarrow{v_{n}^{\prime}} .
\end{aligned}
$$

Here, $v_{r}$ is the relative velocity between $\overrightarrow{v_{l}^{\prime}}$ and $\overrightarrow{v_{n}^{\prime}}$. $Q\left(\overrightarrow{v_{l}}\right)$ is determined using $P$ and it is weighed with the neutral particle velocity distribution function $f_{n}\left(\overrightarrow{v_{n}}\right)$, which is assumed to have an isotropic Maxwellian distribution. $f_{i}\left(\overrightarrow{v_{l}^{\prime}}\right)=n_{i} \delta\left(\overrightarrow{u_{x}}-\overrightarrow{v_{l}^{\prime}}\right)$ is the velocity distribution function of the ions. Monte Carlo integration was used for $Q\left(\vec{v}_{l}\right)$. Figure 5 shows $Q\left(\vec{v}_{l}\right)$ integrated over the $z$ direction for the casein which both the ion energy and neutral particle temperature $T_{n}$ are $10 \mathrm{eV}$. The ions fly from one control volume to another control volume. $Q\left(\vec{v}_{l}\right)$ has the same dimensions as the Boltzmann collision operator; thus, its integral in the velocity space becomes a source term for a control volume at position $\overrightarrow{v_{l}} \times d t$, as shown in Fig. 6. The density flowing out from the control volume is subtracted. The sum becomes the source term for each control volume. For example, the source terms for the control volume $J$ are written as follows: 


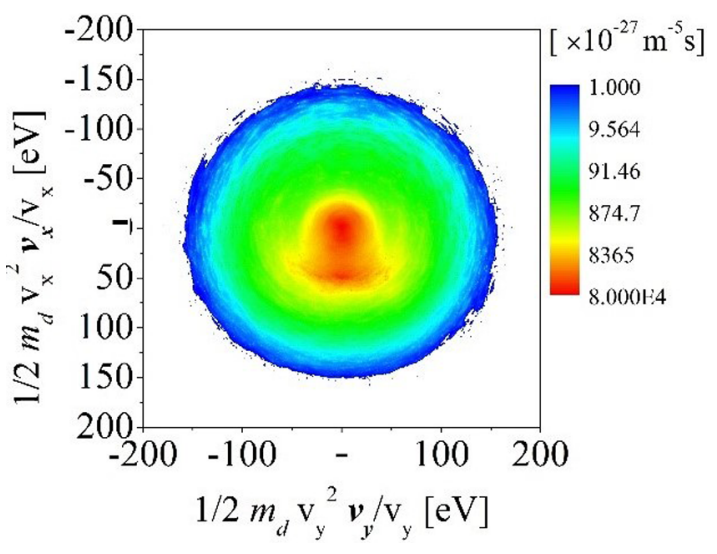

Fig. 5 Example of $\theta\left(\overrightarrow{v_{l}}\right)$ integrated over the $z$ direction when the $10 \mathrm{eV}$ ions are scattered by neutral particles with $10 \mathrm{eV}$ Maxwellian distribution.

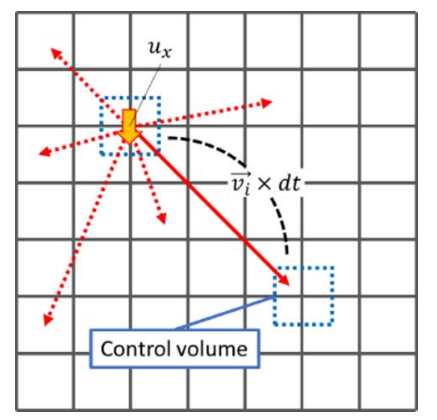

Fig. 6 The ions scattered by neutral particles fly from one control volume to another control volume.

$$
\begin{aligned}
S_{p_{E L}}^{J}= & \sum_{k_{1}=1\left(k_{1} \neq J\right)}^{m e s h} \int_{V_{k_{1} \rightarrow J}} Q_{k_{1}}\left(\overrightarrow{v_{l}}\right) d \overrightarrow{v_{l}} \\
& -\sum_{k_{1}=1\left(k_{2} \neq J\right)}^{m e s h} \int_{V_{J \rightarrow k_{2}}} Q_{J}\left(\overrightarrow{v_{l}}\right) d \overrightarrow{v_{l}}, \\
\overrightarrow{S_{P_{E L}}^{J}}= & \sum_{k_{1}=1\left(k_{1} \neq J\right)}^{m e s h} \int_{V_{k_{1} \rightarrow J}} m_{i} \overrightarrow{v_{l}} Q_{k_{1}}\left(\vec{v}_{l}\right) d \overrightarrow{v_{l}} \\
& -n_{i} n_{0}\langle\sigma v\rangle_{E L} \cdot \vec{u}, \\
S_{i_{E L}}^{J}= & \sum_{k_{1}=1\left(k_{1} \neq J\right)}^{m e s h} \int_{V_{k_{1} \rightarrow J}} \frac{1}{2} m_{i} v_{i}^{2} Q_{k_{1}}\left(\vec{v}_{l}\right) d \vec{v}_{l} \\
& -n_{i} n_{0}\langle\sigma v\rangle_{E L} \frac{1}{2} m v_{i}^{2} .
\end{aligned}
$$

\subsection{Parameters of the neutral particles}

The neutral particles density and temperature are assumed to be $n_{n}=\alpha_{n} \times n_{i}$ and $T_{n}=\alpha_{T} \times T_{i}$, respectively, based on the corresponding parameters $\alpha_{n}$ and $\alpha_{T}$. In this study, $\alpha_{n} \sim 0.2-0.4$ and $\alpha_{T} \sim 0.1-0.5$ were used.

\section{Results and Discussion}

\subsection{Effect on the 2-D profile}

The plasma and neutral particles were considered to be only deuteron, electron, and deuterium. Appropriate (a)

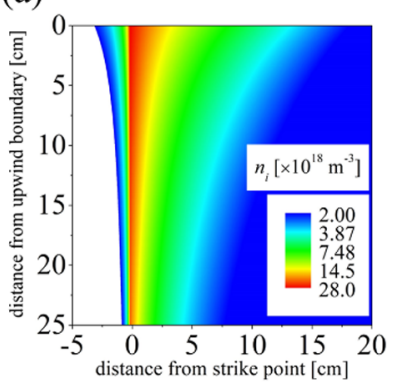

(b)
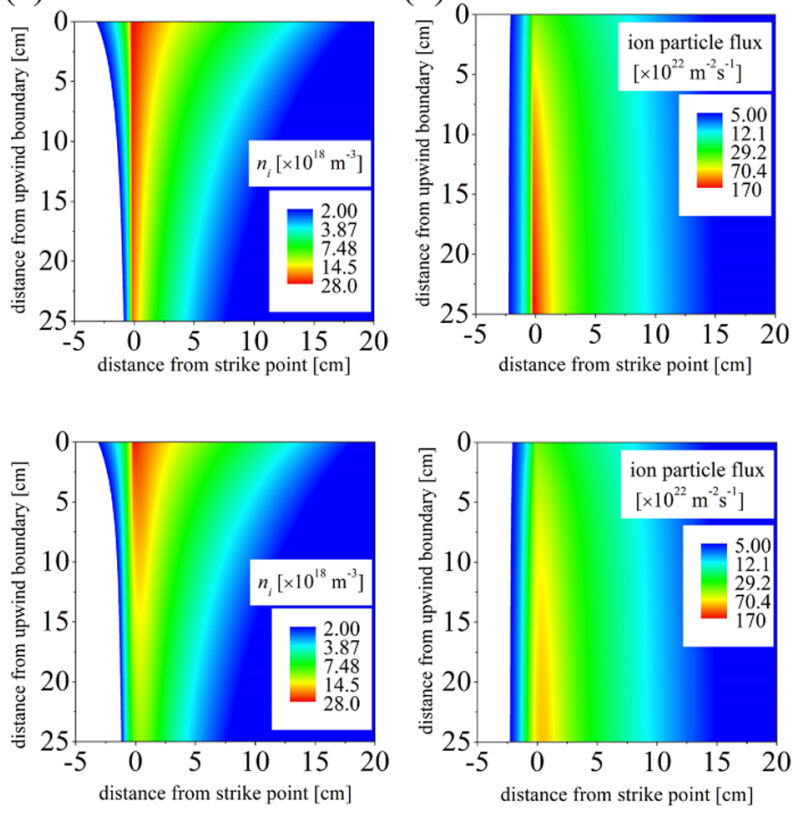

Fig. 7 2-D profile of the (a) plasma density and (b) ion particle flux obtained from the diffusion and EL models.

values for the parameters $\alpha_{n}$ and $\alpha_{T}$ were first searched to reproduce the electron density at the divertor plate by the SONIC code. The values were found to be $\alpha_{n}=0.4$ and $\alpha_{T}=0.3$. The EL model was also simulated with the same parameters. Figures 7 (a) and 7 (b) show the results of the plasma density 2-D profile and ion particle flux obtained from the diffusion model and the EL model, respectively. Each value computed with the EL model is spread in the radial direction, while this is not the case for the diffusion model. In contrast, 2-D profiles of velocity and temperature were not changed significantly. This could be due to the fact that the elastic scattering transport source term is smaller than the other source terms.

\subsection{Effect on the ion density profileatthe divertor plate}

The influence of parameters $\alpha_{n}$ and $\alpha_{T}$ on the density profile at the divertor plate was evaluated. Figure 8 shows the profile of the plasma density at the divertor plate obtained with the diffusion and EL models when $\alpha_{n} \sim 0.2$ 0.4 . In either case, the plasma density near the strike point of the EL model is smaller than that of the diffusion model. Moreover, the density peak is shifted outwards, reflecting a trend that has also been observed in the experiments. As the neutral density becomes larger, the density peak increases, because more ionization events occur.

Figure 9 shows the plasma density profile at the divertor plate obtained with the diffusion and EL models when $\alpha_{T} \sim 0.1-0.5$. In the case of the diffusion model, the change in $\alpha_{T}$ does not significantly affect the profile. When $\alpha_{T}$ is large, the density is spread in the radial direction in the EL model, because ions are scattered more strongly in 


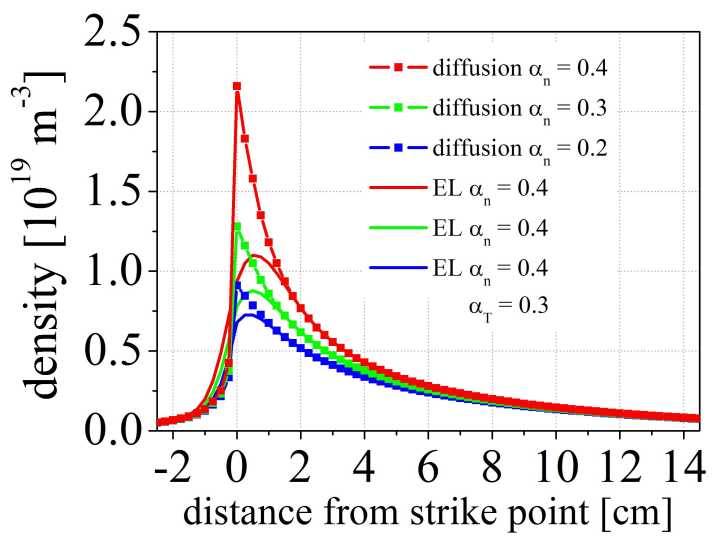

Fig. 8 Comparison of the radial transport effect with different $\alpha_{n}$ values on the ion density profile at the divertor plate.

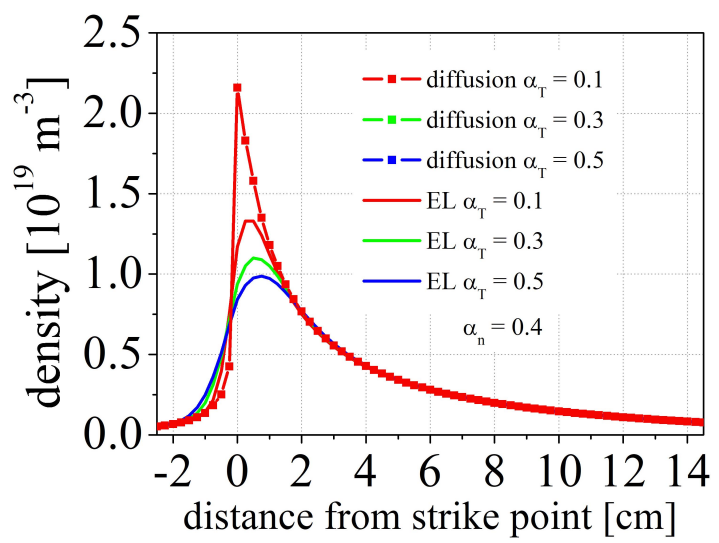

Fig. 9 Comparison of the radial transport effect with different $\alpha_{T}$ values on the ion density profile at the divertor plate.

the high neutral particle temperature space.

On the other hand, there is almost no effect on the electron temperature when using the EL model. The electron temperature has also been observed to spread in the radial direction in the experiments. In this work, no additional term was used for the electron temperature conservation equation. It will be required for the prediction of quantitative electron temperature to improve the present model.

\subsection{Effect on the ion flux peak at the divertor plate}

Figure 10 shows the ratio of the ion flux peak at the divertor plate obtained with the diffusion and EL models when $\alpha_{T} \sim 0.1-0.5$. As the temperature of the neutral particles increases, the ratio of the ion flux decreases. This is due to the fact that, when the neutral particle temperature is high, the ions are scattered strongly. The ion flux peak can be reduced to half of the value predicted by the diffusion model by taking the large-angle elastic scattering transport into consideration. The ratio value corresponds to the ratio between the results of the SONIC code and the experimental value.

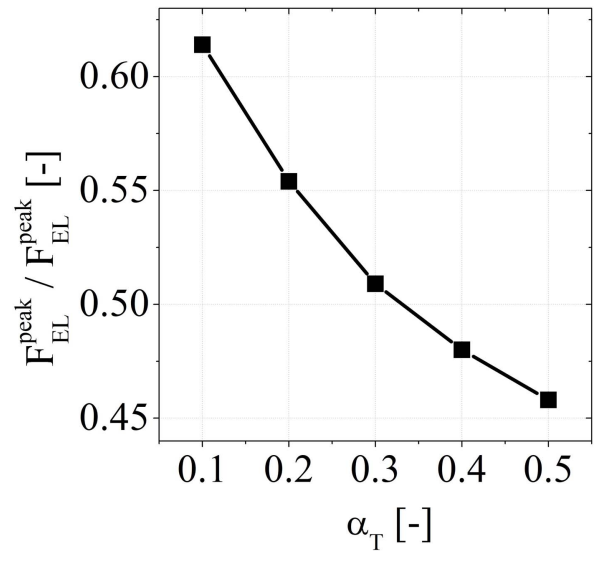

Fig. 10 Relative comparison of the radial transport effect with different $\alpha_{T}$ values on the ion flux peak at the divertor plate.

\section{Conclusions}

A transport model driven by the large-angle elastic scattering between ions and neutral particles was proposed. This model may be able to describe the disagreement between simulations and experiments. The 2-D profile of the plasma density and ion flux were found to be spread more extensively in the radial direction using the EL model rather than by the diffusion model. The plasma density peak was reduced to about half of that of the diffusion model.

In contrast, almost no effect was observed on the ion and electron temperature. The electron temperature was also found to spread in the radial direction in the experiments. In this study, no new term was introduced in the source term of the electron energy conservation equation. Further model improvements, including a new term for the electron equation, are needed. In addition, the model needs to be validated by applying the integrated codes.

[1] A.S. Kukushkin et al., Fusion Eng. Des. 86, 2865 (2011).

[2] S. Wiesen et al., J. Nucl. Mater. 463, 480 (2015).

[3] X. Bonnin et al., Plasma Fusion Res. 11, 1403102 (2016).

[4] H. Kawashima et al., Plasma Fusion Res. 1, 031 (2006).

[5] K. Shimizu et al., Nucl. Fusion 49, 3403070 (2009).

[6] A. Taroni et al., Contrib. Plasma Phys. 32, 438 (1992).

[7] T.D. Rognlien et al., Contrib. Plasma Phys. 34, 362 (1994).

[8] Y. Feng et al., J. Nucl. Mater. 266, 812 (1999).

[9] K. Hoshino et al., J. Nucl. Mater. 463, 573 (2015).

[10] S. Baschetti et al., Nucl. Mater. Energy 19, 200 (2019).

[11] T. Takizuka et al., JAERI-Research 2003-010.

[12] P.S. Kristié and D.R. Schultz, At. Plasma-Mat. Interact. Data Fusion 8, 1 (1992).

[13] H. Matsuura and Y. Nakao, Phys. Plasmas 13, 062507 (2006).

[14] H. Matsuura et al., Plasma Fusion Res. 11, 1403105 (2016).

[15] B.J. Braams, NET Rep. 68 EURFU/XII-80/87/68, CEC, Brussels (1987).

[16] S.I. Braginskii, Trans. Processes Plasma Rev. of Plasma Phys. 1, 205 (1965).

[17] R. Schnelder et al., Contrib. Plasma Phys. 461 (2006). 